\title{
Prager Geschichte, Weltgeschichte und (inter-)kulturelle Identität: \\ Ota Filips Roman Café Slavia als Gegenstand der Auslandsgermanistik
}

\section{Julia Enzinger}

In Café Slavia the Moravian author Ota Filip portrays the history of Prague as a setting of world history and criticizes the process of coming to terms with it. By telling the story of an Austrian Aristocrat and his struggling search for identity in Prague's multicultural society, Filip discusses the ambivalent development of Czech culture and its connection to German culture and history. The first part of the paper offers a literary study of the novel's main points revealing Filip's message of a joint, future-oriented examination of history. In the second part of the paper, describing an intercultural semester project based on Café Slavia (a product of Czech as well as German culture), it will be demonstrated how this message can be implemented in German studies abroad.

Ota Filip - Café Slavia - Czech-German history - history of Prague - Czech culture cultural identity - intercultural literary teaching - literature in German studies abroad - city tour Prague

In Café Slavia stellt der mährische Autor Ota Filip die Geschichte Prags als Schauplatz der Weltgeschichte dar und kritisiert deren Aufarbeitungsprozess. Mit der Lebensgeschichte eines österreichischen Adligen und dessen problematischer Identitätssuche in der multikulturellen Prager Gesellschaft diskutiert Filip die ambivalente Entwicklung der tschechischen Kultur und deren Verbindung zur Kultur und Geschichte Deutschlands. Der erste Teil des Artikels bietet eine literaturwissenschaftliche Untersuchung des Romans, die Filips Botschaft einer gemeinsamen, zukunftsorientierten Aufarbeitung der Geschichte herausarbeitet. Im zweiten Teil wird anhand eines interkulturellen Semesterprojekts zu Café Slavia - als einem Produkt sowohl der tschechischen als auch der deutschen Kultur - gezeigt, wie diese Botschaft in der Auslandsgermanistik umgesetzt werden kann.

Ota Filip - Café Slavia - tschechisch-deutsche Geschichte - Geschichte Prags - tschechische Kultur - kulturelle Identität - interkulturelle Literaturvermittlung - Literatur in der Auslandsgermanistik - Stadtführung Prag

\section{Ota Filips Literatur zwischen Tschechien und Deutschland, Vergangenheit und Zukunft}

„Ich habe von der großen Geschichte die Schnauze voll." So äußert sich Ota Filip 2012 in der ersten Vorlesung seiner Reihe Verspätete Abrechnungen (Filip 2012: 9). Diese Aussage verwundert zunächst, ist die Geschichte doch eines der zentralen Themen in Filips Werken, insbesondere in seinem ersten deutschsprachigen Roman 
Café Slavia. ${ }^{1}$ Ein kurzer Blick auf Filips Biografie gibt jedoch eine Erklärung für dessen Verdruss.

Filip wächst als Sohn eines Deutsch-Tschechen und einer Polin in den 1930ern und 1940ern in Schlesisch Ostrau auf, das bis 1939 Teil der Tschechoslowakischen Republik und anschließend Teil des Großdeutschen Reichs ist. ${ }^{2}$ Nach dem Ende des Zweiten Weltkriegs zieht Filip nach Prag, das nun unter dem kommunistischen Regime steht, studiert dort und beginnt als Sport-Redakteur zu arbeiten. 1951 muss er wegen „bourgeoiser Gesinnung“ (Filip 2012: 119) zum Militärdienst antreten, wird 1959 aus demselben Grund aus der Kommunistischen Partei ausgeschlossen und schließlich bis 1968 zur Zwangsarbeit verurteilt. Während dieser Zeit beginnt Filip trotz Schreibverbot die Arbeit an seinem ersten Roman, Cesta ke hřbitovu. Mit dem Prager Frühling kehrt er als Verlagslektor nach Ostrau zurück, wird dort jedoch wegen Systemkritik für achtzehn Monate in Haft geschickt und ab 1970 erneut zur Zwangsarbeit verurteilt. 1974 geht Filip dann ins Exil nach Westdeutschland, wo er fortan auf Deutsch publiziert - unter anderem den Roman Café Slavia - und bis zu seinem Tod im März 2018 wohnhaft bleibt.

Angesichts dieser ereignisreichen Biografie erklärt sich, warum Filip genug hat von "der großen Geschichte" (Filip 2012: 9), die er als Zeit politischer Umstürze und „sich selbst als gerecht lobende[r] Regime“ (Filip 2012: 9) erlebt. Dass er diese dennoch immer wieder zum Hauptthema seiner Werke macht, begründet er mit folgender Beobachtung:

„Wir, die Tschechen und Mährer, haben nämlich [...] eine sonderbare Eigenschaft: Wenn wir in unserer Geschichte - sehr oft durch unser eigenes Verschulden - in einen politischen Schlamassel oder in eine Katastrophe gerieten [...], dann versuchten wir nie, unsere Krisen aus eigener Kraft zu bewältigen und zu überwinden“ (Filip 2012: 33).

In seinen Romanen setzt sich Filip mit der tschechischen Geschichte auseinander, um einerseits ihre Aufarbeitung voranzutreiben, andererseits um „den freien Geist [...] für die Zukunft zu bewahren“ (Filip 2012: 43f.). Inwiefern sein ExilRoman Café Slavia diese Implikationen aufweist, wird im ersten Teil des Beitrags untersucht. Der zweite Teil veranschaulicht anhand eines Unterrichtskonzepts, welches Potenzial Filips Roman für die tschechische Auslandsgermanistik birgt, zumal die Beschäftigung mit dem gebürtigen Ostrauer in dessen Todesjahr besonders angebracht scheint. In Form einer Projektskizze für eine literarische Stadtführung durch Prag wird erläutert, wie sich im Rahmen eines interkulturellen

1 Die Erstauflage erschien 1985 bei S. Fischer, Frankfurt a. M. und liegt der hier verwendeten Edition zugrunde: Filip, Ota (2001): Café Slavia. München, Herbig. Diese wird zur Zitation im Folgenden abgekürzt mit dem Sigel „CS“.

2 Die folgenden biografischen Informationen entstammen allesamt der Vita, die Filip seinen Vorlesungen in Verspätete Abrechnungen angehängt hat. Vgl. hierzu Filip (2012: 119-120).

Brünner Hefte zu Deutsch als Fremdsprache • Jahrgang 11・Nummer $1 \bullet 2018$ 
DaF-Unterrichts literaturwissenschaftliche und geschichtliche Lerninhalte mit sprachlichen verbinden lassen.

\section{Literaturwissenschaftliche Textanalyse der zentralen Romanthemen und -motive}

\subsection{Graf Belecredos zwischen Identitätsbildung und -verlust}

\subsubsection{Der Maskenwechsel als Motiv der Identitätsdiffusion}

Identität ist eines der zentralen Themen, mit denen sich Ota Filip auseinandersetzt - sowohl in seinen Fiktionen als auch in der Wirklichkeit. Auf die Frage nach seiner Herkunft antwortet der Literat: „Ich bin ein zeitgenössischer Nomade zwischen zwei europäischen Sprachen und zwei europäischen Kulturen“ (Filip 2012: 63). Er sieht sich als Mitglied sowohl der deutschen als auch der tschechischen (Sprach-)Kultur und verortet sich in einer Zwischenposition, die auch auf seinen Protagonisten in Café Slavia, den Grafen Belecredos, zutrifft.

In Café Slavia schildert Belecredos, ein Angehöriger einer alten österreichischen Adelsfamilie, einem Spaziergänger seine Lebensgeschichte, die sich im Prag des 20. Jahrhunderts abspielt. In seinen Erzählungen vermischt der Graf Privates mit öffentlich-politischen Ereignissen. Seine Familienhistorie beginnt 1620 mit der Schlacht am Weißen Berg, bei der „der erste Belecredos siegreich nach Prag eingezogen war" (CS: 132), und führt über die zwei Weltkriege und das kommunistische Regime hin zum Ende des Prager Frühlings 1968, das auch sein persönliches Ende bedeutet (vgl. CS: 266f.). Bereits hier zeigt sich eine Parallele zu Filips Leben: das Miterleben dessen, was heute als Geschichte bezeichnet wird. Wenngleich der Graf das Ende der Habsburgermonarchie erlebt, Ota Filip hingegen erst 1930 geboren wird, so sind doch beide Zeitzeugen der großen politischen Umwälzungen, die Prag bzw. Tschechien im 20. Jahrhundert erfährt. ${ }^{1}$

Eine weitere Gemeinsamkeit besteht in der kulturellen Zwischenposition. Während Filip als gebürtiger Tscheche nach Deutschland flieht und schließlich ein „Zuhause in der deutsch-bayerischen Fremde" findet (Filip 2012: 64), scheint es, dass sich Belecredos nie beheimatet fühlt, ja dass er sich selbst zum Fremdling in seiner Heimat macht. Als österreichischer Graf in Prag ist Belecredos ebenfalls Mitglied zweier Kulturen. Doch sein Maskenspiel deutet daraufhin, dass er sich keiner Kultur gänzlich anschließen kann oder will: Der Graf scheitert an der Frage nach der eigenen Identität.

Schon zu Beginn der Erzählung erklärt Belecredos, er trage seit einer Verletzung in jungen Jahren täglich eine andere Masken samt Kostüm, sodass er ,jeden Tag

1 Der Verbindung von Autobiografie und Zeitgeschichte geht auch Jan Kubica nach, der in seiner Monografie Spisovatel Ota Filip (2012) dessen literarisches und journalistisches Wirken sowohl historisch-politisch als auch sprachlich-kulturell kontextualisiert. 
ein anderer Mensch“(CS: 9) sei. Zu den Maskierungen zählen beispielsweise der „kesse Bohemian“ (CS: 10), der „schwermütige, melancholische Herr aus besseren Kreisen“ (CS: 11) oder ein „ganz gewöhnlicher Passant mit gelangweiltem Ausdruck" (CS: 11). Er tauscht Kleider, Namen und Nationalitäten aus, nimmt fremde Persönlichkeiten an - beispielsweise die Wenzels von Salmo (vgl. CS: 264) oder die Lenins (vgl. CS: 267) - und verändert damit nicht nur sein Aussehen, sondern wechselt seine Identität. Was der Graf damit letztlich erreicht, ist keine „Selbstfindung in einer Identitätsmultiplität“ (Kliems 2002: 199), wie man sie für seinen literarischen Schöpfer Filip feststellen kann, sondern die Konstruktion einer künstlichen Identität, die zur Selbsttäuschung wird. Oder um mit den Worten des Erzählers zu sprechen: „Der Mensch kann seine Kleider wechseln und Masken aufsetzen, damit ist es aber mit seiner Veränderung noch nicht getan“ (CS: 9).

In ihrer Untersuchung zum Thema „Identität in der osteuropäischen Exilliteratur“ hält Kliems fest, dass die Maskierung ein gängiges Motiv der (De-)Figuration darstellt (vgl. 2002: 220). 1 Im Falle des Grafen Belecredos hingegen kommt es durch die Masken weniger zur „Gesichtbildung“ (Kliems 2002: 220) eines Romanhelden als vielmehr zur Identitätsdiffusion eines Antihelden. Belecredos ist bemüht, sich äußerlich an die jeweiligen politischen und gesellschaftlichen Bedingungen anzupassen. Doch sobald er seine Masken ablegt, sieht er eine „zerfallene Landschaft nach einem permanenten Erdbeben“ (CS: 26). Er erkennt, dass sein Masken- und Namenwechsel nichts weiter als ein Akt der Selbstverfremdung ist, dem er letzten Endes zum Opfer fällt, wenn es da heißt:

„Tausend bekannte, halb oder ganz im Gedächtnis vergrabene Namen, die ich einmal getroffen, mir ausgedacht oder zugelegt hatte, surren, summen, [...] sausen oder stehen still rund um meinen kahlen Kopf" (CS: 26).

Was bleibt ist sein kahler Kopf, ein entleertes, verwirrtes Ich, das sich selbst zu zerstören droht. Denn die Protektion, die sich der Graf anfänglich von seinen Masken, seinem „Harnisch“ (CS: 239), erhofft, geht zusehends verloren: „Ich werde immer durchsichtiger und zerbrechlicher. Ein einziger Schlag würde ausreichen, um mich zu zersplittern“ (CS: 212). Dieser Schlag trifft ihn schließlich, als er vom kommunistischen Regime wegen reaktionärem Verhalten angeklagt wird und sich maskenlos vor Gericht verantworten muss (vgl. CS: 248). Ohne Verkleidung fühlt er sich derart „nackt und fröstelnd“ (CS: 248), dass er am Ende sogar maskiert aus dem Leben tritt (vgl. CS: 267).

1 Zur Darstellung von Identität und Fremd- bzw. Andersartigkeit (u. a.) in der tschechischen und deutschen Literatur siehe auch die von Sandra Kersten und Elke Schenke herausgegebene Sammlung Spiegelungen (2005). 
Das Motiv des Maskenwechsels verweist hier auf die Bildung einer Schein-Identität, hinter der sich Belecredos vor der Wirklichkeit zu schützen sucht. Diese Form des Mitläufertums verwehrt es dem Grafen, sein Ich mit der Wirklichkeit in Einklang zu bringen, denn jeder neue Bezugsrahmen bedarf eines neuen Kostüms. Mit dem immer größer werdenden Maskendepot steigt die Selbst-Verwirrung, während die erhoffte Schutzfunktion zunehmend schwindet. Doch anstatt sich der Realität zu stellen, wendet sich der Verwandlungskünstler einer weiteren Schutzmaßnahme zu: der Flucht ins Café Slavia.

\subsubsection{Das Café Slavia: Ein "sicherer Hafen“?}

Das Café Slavia ist der scheinbar einzige Ort, der dem Grafen Sicherheit gibt. Schon bei dessen erster Erwähnung betont der Graf „Glanz, Wärme und Geborgenheit“ (CS: 44) des Cafés, nennt es mehrfach seinen „sicheren Hafen“ (CS: 224) oder „Ankerplatz“ (CS: 83). Die heimatlichen Gefühle, die er für das Café entwickelt, wirken zunächst nachvollziehbar, handelt es sich dabei doch sowohl im Roman als auch in der Realität um ein Kaffeehaus, das vor allem für seine „noble Kundschaft “ bekannt ist.

Seit 1884 gilt das „Luxus-Café“ (Historie 2017) im Zentrum Prags als Treffpunkt für Künstler, Intellektuelle, Personen des öffentlichen Lebens und Adlige zumindest bis zum Ende der Habsburgermonarchie (vgl. Historie 2017). Als letzter Angehöriger einer tausend Jahre alten österreichischen Adelsfamilie entspricht Belecredos der Klientel und wird von Oberkellner Alois sofort als künftiger Stammgast begrüßt (vgl. CS: 83). Die herzliche Begrüßung, die Deklarierung des Cafés als "Zufluchtsort“ (CS: 84) und die Versicherung der Autorität, die Belecredos in den Kreisen des Cafés innehat - „Man kennt Sie in Prag“ (CS: 82), „Sie sind ein Meister" (CS: 152) - machen das Café zu einer Art Heimat für ihn, in der zumindest seiner ursprünglichen, adligen Identität Stabilität gewährt wird. Demgemäß entwickelt der Graf ein strenges Ritual, bei dem er täglich kurz vor dem Mittagsläuten das Café betritt und sich an seinem Platz am Fenster Kaffee und Kognak einverleibt (vgl. CS: 152). Sowohl Belecredos' als auch Alois' Handlungen sind dabei derart eingespielt, dass es dem Grafen unmöglich ist, „die Jahre im Café Slavia zu unterscheiden“ (CS: 152). Tag für Tag sitzt er am Fenster und beobachtet die Geschehnisse außerhalb bzw. „wie wieder einmal mit Böhmen Geschichte gemacht wurde“ (CS: 163). Er sieht „Demonstrationen, Umzüge, Staatsbegräbnisse“ (CS: 161) und "fremde Armeen" (CS: 161) vorbeiziehen, doch seinen Ritualen bleibt er selbst in Kriegszeiten treu (vgl. CS: 170, 213). Dem wechselhaften Verlauf der Geschichte setzt der Graf die Stabilität seiner Café-Heimat entgegen. Letztlich

1 Historie Café Slavia. auf: https://www.cafeslavia.cz/de/uber-uns/historie/ (16.08.2017). 
scheitert aber auch dieser Versuch, einen Zufluchtsort und eine konstante Identität zu etablieren.

Denn Belecredos gilt den anderen Gästen aufgrund seiner Ritualträchtigkeit zwar als „letzter Orientierungspunkt in der verworrenen Zeit" (CS: 213), doch betont er immer wieder seine Einsamkeit (vgl. CS: 152, 163). Dass er dafür selbst verantwortlich ist, scheint er nicht zu erkennen. Der Graf bleibt stets nur Beobachter des Zeitgeschehens, „wie ein Gespenst, [...] ein Zuschauer in der Prominentenloge eines sonst leeren Theaters" (CS: 171), ohne daran teilzunehmen. Als „monarchistisches Kaffeehaus-Individuum“ (Kliems 2002: 223) schottet er sich sowohl von seinen Mitmenschen als auch von der Realität ab. Das Café Slavia, das auf den ersten Blick eine identitätsstiftende Funktion in einer angesehenen Position hat, ist zugleich verantwortlich für seinen Ausschluss aus der gesellschaftlichen Wirklichkeit.

In diesem Zusammenhang verweist Kliems zu Recht auf die Nähe zu dem in der tschechischen Literatur gängigen hospoda-Topos, der hier einer Verfremdung unterzogen wird (vgl. 2002: 222f.). Der tschechische Begriff hospoda steht für ,Wirtshaus', ,Gastwirtschaft ${ }^{1}$ und wird in der Literatur zur Inszenierung eines gemeinschaftlichen Treffpunkts mit Kontaktfunktion, einer „Enklave öffentlicher tschechischer Kommunikation“ (Kliems 2002: 222), verwendet. Im Mittelpunkt steht dabei das hospoda-Ich, eine zentrale Figur, die die Gemeinschaft durch „unentwegtes Reden, Philosophieren und Erzählen" (Kliems 2002: 223) unterhält und über diese gesellschaftliche Funktion ihre eigene soziokulturelle Identität sichert. ${ }^{2}$ Der hospodaTopos stellt also eine Form der Identitätsbildung innerhalb einer Gemeinschaft dar, die auf sozialer Interaktion beruht.

Filips Graf hingegen ist nicht an einer solchen Gemeinschaft interessiert. Zwar philosophiert auch er gerne im Café Slavia, meist geschieht dies aber in Gedanken oder richtet sich allein an Kellner Alois. Dieser grenzt das Café auch dezidiert von gemeinschaftlichen Kommunikationsräumen ab, indem er klarstellt: „Wir sind kein Café der Schwätzer, bei uns wird geschwiegen" (CS: 84). Da Belecredos das Café dennoch - oder gerade deswegen - zu einem Zufluchtsort macht, steht fest, dass die Identitätsbildung nach dem hospoda-Schema bei Belecredos nicht greifen kann, ja gar das Gegenteil bewirkt. Der Graf hat nicht Teil am soziokulturellen Austausch, sondern schließt sich aus. Der ,sichere Hafen' grenzt ihn zweifach von der Wirklichkeit bzw. der Gesellschaft ab: zum einen durch die Zurückgezogenheit innerhalb des Cafés, zum anderen durch die Abkapselung von der Außenwelt.

Hinzu kommt das Geständnis seines vermeintlichen Helfers Alois, der sich unerwartet als Verräter entpuppt. Wie er erklärt, wurde ihm das Dasein als

1 Übersetzung gemäß dem Langenscheidt Digital Wörterbuch Tschechisch - Deutsch.

2 Kliems verweist hier vor allem auf die ,im vorstädtischen Raum verankerten Gasthaus-Figuren“ (2002: 223) in den Romanen von Bohumil Hrabal, Jáchym Topol und Jan Novák (vgl. 2002: 223).

Brünner Hefte zu Deutsch als Fremdsprache • Jahrgang 11・Nummer 1 • 2018 
Belecredos” „Beichtstuhl“ (CS: 246) zuwider und er bekennt: „Ich habe Sie ein halbes Jahrhundert denunziert, Herr Graf. Immer war jemand da, der alles über sie wissen wollte" (CS: 246), was zu einer Verhaftung des Grafen führt. Mit dem Vertrauensbruch des Oberkellners erweist sich die ganze mit dem Café verbundene Stabilität und Kontinuität als Täuschung. Als sich Belecredos dessen nach seiner Freilassung bewusst wird, ist es schon zu spät. Das Café wirkt plötzlich „kalt und düster" (CS: 256), er fühlt sich „niedergedrückt und enttäuscht“ (CS: 256) und gesteht sich ein, „dass unsere Geschichte und die vom Café Slavia zu Ende ist“ (CS: 256).

Schlussendlich erkennt Belecredos, dass er mit seinem Maskenspiel und der künstlichen Café-Persönlichkeit an der Ausbildung der eigenen Identität scheitert. Er droht dorthin zu gelangen, wo er nie hin wollte: in ein „Loch des Vergessens“ (CS: 266). Die einzige Möglichkeit, dem entgegenzuwirken, sieht er in der Verbreitung seiner Lebensgeschichte, weshalb er den Spaziergänger vor seinem Tod darum bittet: „[E]rzählen Sie meine Geschichte, aber vergessen Sie nicht, dass die Wahrheit viel schlimmer ist, als die gräßlichste Lüge“ (CS: 266). Worauf der Graf hier anspielt, zeigt die Erschließung seines Geschichtsbilds.

\subsection{Prager Geschichte als Abbild der Weltgeschichte}

\subsubsection{Prag als Kulisse der Weltgeschichte}

Wie schon erwähnt, behält der Graf auch in Krisenzeiten seine Café-Rituale bei. Trotz militärischer Einmärsche und kriegerischer Auseinandersetzungen verweilt er an seinem Stammplatz und bleibt lediglich Zuschauer des Geschehens. Er beobachtet den Ersten und Zweiten Weltkrieg (vgl. CS: 129f., 170f.), die Übernahme Prags durch das kommunistische Regime (vgl. CS: 186) und schließlich den Prager Frühling (vgl. CS: 252ff.). Dass diese Flucht vor der Realität keine Lösung darstellt, zeigt sich spätestens mit dem Tod des Grafen: Er stirbt, wie sämtliche seiner Vorfahren (vgl. CS: 66), im Keller des Familien-Palais - eingemauert mit einer Maske Lenins auf dem Gesicht (vgl. CS: 267).

Belecredos' Masken fungieren weniger als Schutz denn als Spiegel des Geschichtsverlaufs. Mit deren Wechsel passt sich der Graf stets der politischen Situation an, ebenso wie seine Ahnen, von denen er die Verkleidungen zum Teil ererbt hat (vgl. CS: 102). An dem Tag, an dem der Zweite Weltkrieg endet, legt er zum Beispiel das Kostüm eines älteren Herren mit Kriegsverletzungen an, die dieser „nicht mehr zu verdecken versucht, sondern [...] mit ein wenig Charme zur Schau trägt" (CS: 178). 1946 maskiert er sich als Lenin, um für ein Porträt im Auftrag des sozialistischen Zentralkomitees Modell zu stehen (vgl. CS: 204). Der Graf hat auch 
keinerlei Skrupel, mit dieser Maske wochenlang seinem Café-Ritual nachzugehen, bis sich eine ältere Dame empört: „Ein Graf läuft zu den Kommunisten über, das ist zu viel!“ (CS: 206). Mit dieser Aktion und Vorfällen ähnlicher Art führt Belecredos den maskenhaften Charakter vor, den er dem Verlauf der Geschichte unterstellt. Denn seiner Meinung nach stellt die Weltgeschichte nichts anderes dar, als ein Theater, eine „Komödie“ (CS: 167), in der Prag die Funktion einer Kulisse hat.

Das Geschichtsbild eines Welttheaters verkündet der Graf recht offen, wenn er in Bezug auf die vielen Regimewechsel, die er erlebt, urteilt:

„Ab und zu wurden Kostüme gewechselt, neue Masken angelegt, neue Programme und Ideologien verkündet, aber sie waren hier immer nur das, was sie seit eh und je tatsächlich waren: Metaphern, manchmal ein Grund zu berauschender Euphorie, ein andermal wieder eine absurde Entgleisung der Vernunft" (CS: 161)

Belecredos nimmt politische Umbrüche nicht als gravierende Einschnitte wahr. Aufgrund des theaterhaften Charakters, den er ihnen zuschreibt, werden sie ihm zu nicht mehr ernstzunehmenden Nebensächlichkeiten. Diese Auffassung spiegelt sich in der Art und Weise wider, in der er erzählt. Nicht nur, dass der Graf historische Ereignisse durchweg mit privaten vermischt, stets stellt er - relational betrachtet - Banalitäten in den Vordergrund, während Weltbewegendes nur knapp eingeworfen oder nebensächlich erwähnt wird. Vom Ende des Ersten Weltkrieges und der Gründung der Tschechoslowakei erfährt der Leser zum Beispiel nur durch die Erwähnung der tschechoslowakischen Exilregierung seitens eines Herrn Tkaczyks (vgl. CS: 129f.). Belecredos interessiert sich kaum für die (inter-)nationale Bedeutung des Geschehens, wohl aber für die Auswirkungen auf ihn selbst: den anstehenden Verkauf seines Palais als Botschaftsgebäude der chinesischen Regierung (vgl. CS: 130-32).

Ein weitere beispielhafte Episode ereignet sich im September 1937: Während vor dem Café der Trauerzug für den ersten tschechoslowakischen Staatspräsidenten Tomáš Garrigue Masaryk vorbeizieht, widmet der Graf seine Aufmerksamkeit lieber der Vermietung seines Fensterplatzes an eine zutiefst bewegte Mitbürgerin (vgl. CS: 161). Belecredos zeigt keinerlei Emotionen, für ihn hat das Ereignis als Teil einer sich ständig wiederholenden Geschichte keine Bedeutung, es gibt „kein Ende und keinen Anfang" (CS: 163). Auch der Einmarsch der Nationalsozialisten bringt ihn nicht aus der Ruhe: „Der Vorhang geht für kurze Zeit herunter, Kulissen werden umgebaut, Klamotten gewechselt, neue Fratzen angelegt, Vorhang auf? Und weiter läuft die Komödie!“ (CS: 163f.).

Daneben mokiert sich Belecredos über den regimeabhängigen Namenswechsel von öffentlichen Straßen und Plätzen, z.B. der Brücke der Legionen, „die einmal Franzensbrücke geheißen hatte, später dann den Namen Adolf Hitlers trug, die Sie 
jedoch [...], aus mir unverständlichen Gründen die Brücke des 1. Mai nennen“ (CS: 151). Gleiches gilt für den Moldaukai, „heute Smetana-Ufer, damals [...] Franzenskai, er hatte jedoch [...] so oft den Namen gewechselt, dass ich einige schon längst vergessen habe" (CS: 81). Die politisch bedingten Umbenennungen sind für Belecredos unwesentlich, weswegen er zumeist die ursprünglichen Namen verwendet (vgl. CS: 151f.).

Den Prager Denkmälern wird ein ähnliches Urteil zuteil. Im Kontext des komödienhaften Geschichtsverlaufs fungieren sie sozusagen als Requisiten, weswegen der Graf sie nicht würdigen kann - zumindest nicht in ihrer Funktion als Ehrenmale oder Erinnerungsorte. Zwar reflektiert er bei seinen Spaziergängen stets deren geschichtlichen Hintergrund, einen historischen Wert spricht er ihnen aber ab. Stattdessen nutzt Belecredos die Denkmäler als Anknüpfungspunkte zur (Selbst-)Reflexion, indem er Statuen, wie etwa die des Jan Nepomuk (vgl. CS: 112f.) oder die Karls IV. (vgl. CS: 14f.), zu Zuhörern seiner Anekdoten macht oder sie als Anlass zur Kritik an Kultur und Geschichte nimmt.

\subsubsection{Belecredos' Geschichtsbild: Ein kritischer Blick auf die tschechische Kultur}

Im Kontext der zahlreichen politischen Umbrüche, denen Prag bzw. Tschechien im Lauf der Zeit unterliegen, bemängelt Belecredos das nahezu heuchlerische Verhalten seiner Mitbürger gegenüber den jeweils neuen Machtinstanzen:

„Das ist in Prag immer so, [...] man probt hier Aufstände, man lehnt sich gegen die Mächtigen auf, natürlich jedes Mal mit Begeisterung und auf moralische Überlegenheit gestützt. Aber dann marschieren Armeen ein und aus ist es." (CS: 260)

Der Graf beklagt, dass sich derartige Vorgänge, die mit dem Einmarsch fremder Truppen beginnen und ,in gewaltsamer Befriedung“ (Faryar 2005: 90) enden, in der Geschichte permanent wiederholen und sich die Prager dennoch immer wieder mitreißen lassen, ohne vergangene Rückschläge zu bedenken. Aus diesem Grund bezeichnet er sie als "Versager" (CS: 134), als „betrügerische Weltverbesserer“ (CS: 149) und wirft ihnen vor: „Für jeden Irrtum finden sie stets nachträglich eine Rechtfertigung" (CS: 134), ein Eingeständnis möglicher (Mit-)Schuld fehlt. Für Belecredos mangelt es an einer ehrlichen Auseinandersetzung sowie an einer zukunftsorientierten Aufarbeitung der Geschichte, um diesen Kreislauf zu durchbrechen.

Die nachträglichen Rechtfertigungen hält der Graf nicht nur für verspätet, sondern auch für unaufrichtig. Mit jeder neuen Machtinstanz wechseln die Prager scheinbar unbedacht ihre Ideologie: „Der Glaube an Gott, an politische Programme oder 
ähnliches waren hier nur Metapher" (CS: 134). Politische oder religiöse Einstellungen an sich sind demnach unerheblich, weil sie - ebenso wie die Masken - ständig gewechselt werden und nichts an dem grundsätzlichen Verhalten der Bürger ändern.

Zur Veranschaulichung seiner Vorwürfe greift Belecredos unter anderem die Legende der Heiligen Ludmila auf, mit deren Denkmal er von Zeit zu Zeit Gespräche führt. Diese wurde von ihrer Schwiegertochter, der regierenden Fürstin Böhmens, Drahomíra, aus politischen Gründen ermordet - oder wie der Graf meint: „[D]u standest ihr eben im Weg“ (CS: 237). Die posthume Heiligsprechung der Christin empfindet Belecredos angesichts ihres Schicksals als Heuchelei, ja gar als Betrug, zumal auch der Sohn ihrer Mörderin, Wenzel, später heiliggesprochen wird (vgl. CS: 237). Mit diesem Beispiel verdeutlicht der Graf seine Meinung, nach der die Prager dazu neigen, historische Verbrechen zu beschönigen. Zynisch urteilt er: „Die Mörder und ihre Opfer sind in Legenden eingegangen und leben in der permanent verfälschten Geschichte friedlich nebeneinander" (CS: 237f.).

Mit dieser harschen Kritik stellt Belecredos die tschechische Kultur als eine Kultur dar, die von „Versagen, von Klagen und Rechtfertigungsversuchen“ (Kliems 2002: 221) bestimmt ist und damit ihre eigene Geschichte zu verfälschen droht. Der Ursprung des Versagens liege in den seit Hunderten von Jahren wiederkehrenden Okkupationen - weswegen Belecredos nicht nur mit seinen tschechischen Landsleuten abrechnet, sondern auch mit den deutschen bzw. österreichischen, vor allem mit dem Adel. Denn die Geschichte des Versagens beginnt für ihn mit der Schlacht am Weißen Berg. Seine Vorfahren seien damals in dem Irrglauben in Prag eingezogen, den Tschechen „eine neue Geschichte gebracht zu haben“ (CS: 261). Doch mit diesem Gefecht lösen die Belecredos einen Teufelskreis aus, seit dem jeder Einmarsch nichts weiter als eine Fortführung einer Geschichte der Fremdbestimmung sei.

Dementsprechend legt der Graf den Pragern die Worte in den Mund: „Was alles hätten wir vollbringen können, wie groß und stark hätten wir vor dem Antlitz der Geschichte sein können, wenn wir nicht ständig von fremden Mächten besetzt und regiert worden wären!“ (CS: 261). In diesem „dreihundert Jahre alten böhmischen Gejammer" (CS: 261) sieht Belecredos jedenfalls keine Lösung.

Aufgrund der Beteiligung seiner Vorfahren sieht sich der Graf mitverantwortlich für den folgenschweren Sieg am Weißen Berg (vgl. CS: 261). Bei Faryar heißt es, er sei „selbst ein Täter und macht daraus auch keinen Hehl“ (2005: 171). In der Tat geht Belecredos mit seiner ererbten Teilschuld offen um. Er bezeichnet sich selbst als „Schmarotzer" (CS: 249) und findet es nur fair, dass ihm die familiären Hinterlassenschaften, seine „Güter in Nordböhmen“ (CS: 181), nach dem Zweiten Weltkrieg entzogen werden, denn ,über dreihundert Jahre haben wir aufgrund dieser Erträge gefaulenzt und uns die Bäuche vollgeschlagen" (CS: 181). Ohne Weiteres nimmt er auch die Haftstrafe an, zu der er durch Alois' Verrat verurteilt wird; die 
Amnestie, die im Zuge des Prager Frühlings zuteil wird, will er nicht akzeptieren (vgl. CS: 250-52). Der einzige Ausweg, der ihm angemessen scheint, ist der, sich in Luft aufzulösen: „Es wäre gerecht, wenn ich einmal in lauter Splitter zerfallen würde" (CS: 65).

Bevor dieser Wunsch mit seinem Tod in Erfüllung geht, hält der Graf aber noch an seinem Status fest - zumindest im Café Slavia. Seine Rituale werden zur Protestform: als „Notwehr gegenüber der Repression der Geschichte, Gesellschaftsordnung und staatlichen Machtmaschinerie" (Faryar 2005: 172). Mit der Zeit erkennt Belecredos jedoch, dass sich seine "Saturiertheit und Ritualergebenheit" (Kliems 2002: 223) nicht mit der wechselhaften Geschichte vereinbaren lässt. Auch Alois prophezeit das Ende der österreichischen Adelsprivilegien und rät dem Grafen daher, „vom Café Slavia Abschied zu nehmen“ (CS: 245).

Angesichts dieser düsteren Zukunftsaussichten ist Belecredos' Tod nicht nur die logische Konsequenz seiner gescheiterten Identitätsbildung, sondern vor allem auch die seiner Kultur- und Geschichtskritik: Der Graf mauert sich, als Lenin maskiert, im Keller des ehemaligen Familien-Palais ein (vgl. CS: 267). Zum einen beendet er damit - traditionsgemäß - die 300 Jahre alte Linie der Belecredos, zum anderen erfüllt sich so sein Anliegen, sich spurenlos von der Welt zu verabschieden, wird seine Leiche aufgrund der Maske doch tatsächlich für die Lenins gehalten (vgl. CS: 245f.). Sogar im Tod macht sich der Graf noch über das theaterhafte Geschichtsspiel lustig, sein Ableben fungiert als finaler "Protestakt und [...] tragikomische Karikatur der Geschichte" (Faryar 2005: 173) zugleich, wenn dieses letzte Maskenspiel im Botschaftsgebäude der BRD von dieser als „sowjetisch-tschechoslowakische Provokation" (CS: 267) aufgefasst wird.

\subsubsection{Das Ziel der "Weltverschwägerung“}

Im Zuge seiner Kritik spricht Belecredos von einem „Misthaufen der Geschichte“ (CS: 48), zu dem jeder Einzelne mit seinem persönlichen Abfall, etwa in Form eines unreflektierten Mitläufertums oder der Anwendung von sinnloser Gewalt, beitrage (vgl. CS: 48). Der Boden, „in welchem unsere Saat keimen und wachsen soll“ (CS: 48), sei daher unfruchtbar. Um den Prager Boden wieder fruchtbar zu machen, entwickelt Belecredos die Theorie der Weltverschwägerung. Darnach soll die ganze Welt durch die Zeugung von Kindern „quer zu allen Unterschieden und ideologischen Querelen“ (CS: 122) in ein undurchschaubares Verschwägerungsverhältnis treten. Die so entstehenden Generationen hätten nicht nur ein außerordentlich großes Zusammengehörigkeitsgefühl, sondern seien aufgrund der unklaren Familienzuordnung auch entlastet von der historischen Schuld ihrer Vorfahren (vgl. CS: 122f.). Somit könne man einerseits eine kollektive Aussöhnung mit der 
Vergangenheit vorantreiben, andererseits kriegerische Konflikte vermeiden oder gar „irgendwann in einer stillen, unblutigen Schlacht diese Welt retten“ (CS: 122).

Da sich der Graf selbst mitverantwortlich für die konfliktlastige Geschichte fühlt, beginnt er seine Theorie in die Tat umzusetzen, indem er mit möglichst vielen Frauen verschiedenster nationaler und sozialer Herkunft Nachkommen zeugt: „Mit meinem Samen verwische ich alle Klassen-, Rassen- und Nationalitätenunterschiede, ich schaffe den Keim zu einer unendlich verschwägerten Gesellschaft" (CS: 122). Durch die Verschwägerung hofft Belecredos außerdem selbst, mit der ererbten Schuld seiner Vorfahren zu brechen: „[K] ein verkommener Adliger“ (CS: 122) sei unter seinen Kindern, alle seien gleich, „ein Mensch wie jeder andere“ (CS: 122) und sie werden "gegen das verzauberte Tor des Schicksals pochen" (CS: 123), d.h. den Kreislauf der Versagensgeschichte beenden.

Der Erfolg der Weltverschwägerungstheorie beruht also auf der Kombination zweier Aspekte: Zum einen bedarf es einer möglichst großen und vielfältigen ,Weltfamilie', zum anderen muss diese einen Umgang mit der Vergangenheit pflegen, der den sonst „unfruchtbaren Boden“ (CS: 48) für die Zukunft fruchtbar macht. Wie sich Belecredos diese Zukunft vorstellt, zeigen die neu entstandenen Verwandtschaftsverhältnisse, die er sich in einem Traum vergegenwärtigt: Er ist „Vater! Großvater! Schwager!“ (CS: 217) zugleich, seine Nachkommen sprechen verschiedene Sprachen, „wobei das Tschechische und Deutsche überwogen“ (CS: 217). Zwar herrschen dadurch chaotische Zustände, die sprachliche Vielfalt begrüßt der Graf aber, ebenso wie sein literarischer Schöpfer, Ota Filip.

Dieser befindet sich nach eigener Aussage in einem „sprachlich magischen Kreis innerhalbvonzweihohen, sprachlichen Mauern “(Filip 2012:15). Sowohldiedeutsche als auch die tschechische Sprache sind ihm einerseits vertraut, andererseits fremd (vgl. Filip 2012: 15). Diese Ambivalenz spiegelt sich in Filips „Nichtidentifikation“ (Faryar 2005: 317) mit beiden Kulturen wider. Zwar gehört er beiden an, doch hat er auch die „gemeinsame Bürde der ganzen Last all der Spannungen und Konflikte beider Völker am eigenen Leib [erfahren] " (Faryar 2005: 317) - eine Erfahrung, die er mit seinem Grafen teilt. Die eigene Zwischenposition, die als „Vermittlerrolle zwischen zwei Kulturkreisen“ (Faryar 2005: 318) gilt, drückt Filip in Belecredos' Weltverschwägerungsziel aus. Beide streben nach einem „hoffnungsvollen Fluchtweg in die Zukunft" (CS: 101), sprachliche Unterschiede sollen dabei kein Hindernis sein, sie sind vielmehr ein Gewinn. Denn mit jeder Sprache „öffnet sich für uns [...] eine neue Geschichte, eine neue Kultur, kurzum ein neues Leben!“ (Filip 2012: 73).

Auch wenn der Graf diesen Optimismus zunächst teilt, erkennt er dann doch, dass „Prag [...] für meine ständig wachsende Familie und für mein Schicksal zu klein [wird]. Ich müsste eigentlich von hier ausbrechen, aber wohin?““ (CS: 164). Faryar stellt für Belecredos hier einen „faustischen Weltschmerz“ (2005: 245) fest, 
der ihn angesichts der Ohnmacht gegenüber dem Verlauf der Dinge überkommt (vgl. 2005:245). Dieses Ohnmachtsgefühl, die „Beraubtheit aller praktischen Möglichkeiten in der Gesellschaft“ (Faryar 2005: 237), ist auch Filip bekannt, wie er in einem Interview verrät (vgl. Faryar 2005: 262-64). Dort erklärt er seine Wahrnehmung wiederum mit einem Spezifikum der tschechischen Geschichte: „Im Leben eines jeden Volkes gibt es Augenblicke, in denen es seine Pflicht ist, sich gegen Unrecht zur Wehr zu setzen. Die Tschechen haben das nie getan, und diese Tatsache lastet wie ein Trauma auf ihnen" (Faryar 2005: 263). Vor allem seit 1945 fühlten sich die Tschechen als „Opfer und Sieger in einem“ (Faryar 2005: 264). ${ }^{1}$

Vor diesem Hintergrund einer kontinuierlichen Zwiespältigkeit, die sowohl der tschechischen Kultur als auch Filips (Er-)Leben anhaftet, ist klar, dass in Belecredos' Ziel der Weltverschwägerung auch eine Botschaft Filips steckt. Diese steht im Zeichen der gemeinsamen Aufarbeitung der tschechisch-deutschen Geschichte ${ }^{2}$ und zielt auf die „Verständigung und Vermittlung zwischen beiden Völkern sowie auf die Öffnung von Tschechien nach Europa" (Faryar 2005: 277) ab. So wie sich Belecredos' Nachkommen unabhängig von sprachlichen, sozialen oder politischen Unterschieden gemeinsam um eine bessere Zukunft bemühen sollen, scheint es auch wünschenswert für Filips Leser.

Dass die Mission des Grafen erfolglos bleibt, ist durchaus Teil der Intention. Das Misslingen Belecredos', der sich am Ende bei seinen Kindern dafür entschuldigt, sie in diese Welt gesetzt zu haben - ,ihr alle hättet nämlich eine bessere verdient“ (CS:249) -, erklärt Filip so: „Meine ,Helden“ sind stets Antihelden, die im unerbittlichen Duell mit ihrer Umwelt immer wieder scheitern, auf die Schnauze fallen“ (2012: 91). Doch ebenso zeichnet sie aus, dass sie sich „immer wieder aufrichten [...], immer wieder auf die Suche nach Gerechtigkeit, nach ein wenig Glück und Sicherheit gehen" (Filip 2012: 91). Somit versteht sich Belecredos' Appell an seine Kinder, aus der absurden Welt „das Beste zu machen" (CS: 249) in gleicher Weise als Botschaft Filips.

Ein „Zukunftsappell“, wie ihn Schmitz (2012: 191) Filips Autorschaft zuschreibt, ist also auch in Café Slavia zu finden. Die deutsch-tschechische Geschichte soll nicht nur gemeinsam aufgearbeitet, sondern fortgeführt und „von beiden Seiten neu geschrieben werden" (Schmitz 2012: 195) - lückenlos und mit dem Ziel einer gemeinsamen, mitteleuropäischen Zukunft. Denn als Teil einer solchen sieht sich

1 Zu Thematisierung der „Nachkriegszeit im Werk von Ota Filip“ vgl. Kubicas gleichnamigen Aufsatz in Slezskýsbornik (2013: 107-120).

2 Der Begriff tschechisch-deutsche Geschichte meint hier die Berührungen, Überschneidungen und gemeinsamen Erlebnisse der tschechischen und deutschen Geschichte, wie z.B. Böhmen unter dem Regiment der Habsburgermonarchie, die Besetzung Prags durch die Nationalsozialisten ab 1939 oder die Vertreibung der Sudetendeutschen ab 1945. Zu dem Begriff und entsprechenden historischen Grundlagen vgl. auch Faryar (2005: 248-70). 
Filip, wenn er auf die Frage nach seiner Heimat letztendlich klarstellt: „Ich bin Mitteleuropäer" (Filip 2012: 152).

Als Reaktion auf Filips Schlussworte der Verspäteten Abrechnungen, seiner Hoffnung, „dass mein geschriebenes Wort mich wenigstens um einige Jahrzehnte überlebt“ (2012: 115), wird nun gezeigt, wie die Auslandsgermanistik einen Teil dazu beitragen kann.

\section{Café Slavia in der Auslandsgermanistik: Ein Konzept für eine literarische Stadtführung als interkulturelles Semesterprojekt}

\subsection{Begründung und Lernziele}

In seinem Aufsatz zur Bestimmung der mitteleuropäischen Auslandsgermanistik hält Grucza fest, dass diese zwar eng mit der Inlandsgermanistik verbunden sei, ihr aber aufgrund des besonderen kulturellen Kontextes und den daraus resultierenden spezifischen Bedürfnissen auch eigenständige Aufgaben bzw. Untersuchungsgegenstände zukommen (vgl. 1997: 308-10). Den „eigentlichen Gegenstand“ der Auslandsgermanistik verortet er im jeweiligen „Deutsch vor Ort“, d.h. in der Ver- und Ermittlung von historischen, gegenwärtigen und künftigen „Spuren des Deutschen“ (alle Grucza 1997: 310) einschließlich seines sozialen und kulturgeschichtlichen Kontextes (vgl. Grucza 1997: 310). Als deutschsprachiges Exil-Werk eines gebürtigen Mähren, der sich selbst als Mitteleuropäer bezeichnet, fällt Café Slavia eindeutig in diesen Gegenstandsbereich.

Hinzu kommt, dass das zentrale Romanthema und die Botschaft des Autors der Vermittlungsfunktion der Auslandsgermanistik nachkommen. Die Darstellung der konfliktlastigen deutsch-tschechischen Geschichte sowie die Notwendigkeit ihrer gemeinsamen Aufarbeitung entspricht der „interkulturelle[n] Verständigung“ (Grucza 1997: 310), die im Fokus der Auslandsgermanistik steht. Daneben stellt Café Slavia ein ideales Beispiel dafür dar, inwiefern „[z]eitgeschichtliche Literatur als Bestandteil der Erinnerungskultur" (Widawska 2015: 66) aufgegriffen werden kann. Eine romanbasierte Stadtführung, wie sie hier vorgeschlagen wird, ermöglicht es, über das Medium der Literatur einen neuen Zugang zur eigenen aber auch zur fremden Geschichte und Kultur zu gewinnen, indem sie deren Speicherungs- oder Erinnerungsorte, z.B. in Form von Denkmälern, mit anderen (fiktionalen) Perspektiven verbindet. ${ }^{1}$ Neben Unterschieden sollen dabei vor allem Gemeinsamkeiten entdeckt und reflektiert werden, sodass das Projekt auch einen Beitrag zu einer „Kultur des Friedens“ (Widawska 2015: 141) leisten kann, deren Entwicklung zum Kernbereich einer interkulturellen Germanistik zählt.

1 Zum didaktischen Nutzen von Stadtführungen als Form der „Wissensvermittlung unter der Bedingung von Fremdheit und Fremdsprachigkeit" vgl. Costa (2010: 106-114) und Harren/ Hoffmann zu entsprechenden „Verfahren des Wissenstransfers“ (2010: 151-159). 
Das folgende Semesterkonzept richtet sich an eine tschechische DeutschLernergruppe mit einem Sprachniveau von C1 bis C2, der mit einer literarischen Stadtführung durch Prag eine Alternative zum klassischen Literaturkurs geboten werden soll. Neben der Schärfung des interkulturellen Bewusstseins steht hier - nach Widawskas Konzept der „Textkompetenz als Instrument des Lernens“ (2015: 111) die Koordinierung von Wissens- und Spracherwerb im Mittelpunkt (vgl. Widawska 2015: 119).

Für die Erarbeitung der Stadtführung muss zunächst mithilfe einer Textanalyse eine inhaltliche Grundlage geschaffen werden. Zu berücksichtigen sind hier insbesondere soziokulturelle und historisch-politische Aspekte aber auch der biografische Kontext Filips. Bei der Auswahl der Analyse-Schwerpunkte sollte auch an mögliche Stationen für die Tour gedacht werden. Die Ergebnisse werden dann von den Lernern in der Rolle der Stadtführer an Dritte weitergegeben, es erfolgt also eine Vermittlung durch Weitervermittlung. ${ }^{1}$

Die Zielvorgabe der Weitervermittlung setzt voraus, dass der Roman, gemäß den Prinzipien eines (fächer-)integrativen Unterrichts (vgl. Kepser/Abraham 2016: 157-160), von den Lernern mit seinem Handlungsort und dessen Geschichte in Bezug gesetzt wird. Die Aktualisierung und Kontextualisierung im konkreten Umfeld der Lerner stellt nicht nur eine Motivation für die Auseinandersetzung mit fremdsprachiger Literatur dar, sondern fördert zugleich selbstständiges, wissenschaftliches Arbeiten. ${ }^{2} \mathrm{Zu}$ den Lernzielen zählen dementsprechend die Erweiterung des allgemeinen Leseverständnisses, die Rezeption wissenschaftlicher Texte, die eigenständige Recherche und Auswertung von Sekundärliteratur sowie grundlegende Fertigkeiten in der Literaturanalyse und -kontextualisierung. ${ }^{3}$ Durch die einzelnen Arbeitsschritte zur Erstellung und Planung der Stadtführung wird zudem die Schreibkompetenz sowie die Erstellung von Kurzpräsentationen trainiert. Daneben tragen die Redebeiträge während der Tour als erweiterte Form des „literarischen Gesprächs“ (Kepser/Abraham 2016: 163) zur Verbesserung der Sprechfertigkeit bei.

1 In diesem Zusammenhang sei auf Harren/Hoffmanns Erkenntnisse über die Bedeutung des Zusammenspiels von Erzählen, Gehen und Zeigen für die Informationsvermittlung verwiesen (vgl. 2010: 250-260).

2 Vgl. hierzu das „Prinzip der Selbsttätigkeit“ bei Köck (2005: 352).

$3 \mathrm{Zu}$ letzteren gehören beispielsweise deklaratives wie prozedurales Wissen über Gattungen und Epochen, die Fähigkeit zum ,Verstehen' von Poetik, Form und literarischer Sprache sowie die Fähigkeit zur „Anschlusskommunikation“ (Kepser/Abraham 2016: 169). 


\subsection{Semesterplan und methodische Vorgehensweise}

Die Stadtführung ist als Projektarbeit für insgesamt vierzehn doppelstündige Sitzungen angelegt, die in sieben Arbeitsphasen gegliedert werden. ${ }^{1}$ Die Phasen eins bis vier widmen sich der Erschließung des Romans unter Berücksichtigung der Biografie des Autors, des Entstehungskontexts und der Verortung im Gesamtwerk.

In der Einstiegsphase sollen die Lerner in zwei Sitzungen einen Überblick über das Leben und Werk Filips erhalten. Nach einer Einführung mit zentralen biografischen Informationen bietet es sich an, die Lerner im Kurs gruppenweise Kurzpräsentationen in Plakatform anfertigen zu lassen. ${ }^{2}$ Angesichts Filips ereignisreichen Lebenslaufs kann man den Gruppen je eine der drei Lebensstationen - Ostrau, Prag und München (vgl. Filip 2012: 119-20) - zuteilen, zu der sie ein Kurzreferat von etwa fünf bis zehn Minuten über berufliche, literarische oder auch politische Tätigkeiten erstellen und dabei Bezug zum jeweiligen (sozial-)politischen Kontext nehmen. Die Recherche kann frei erfolgen oder unter Vorgabe von Quellen, wie zum Beispiel Filips Homepage ${ }^{3}$ oder die autobiografisch geprägten Vorlesungen Verspätete Abrechnungen, die in jedem Fall als Hilfsmaterial genannt werden sollten. Mit Blick auf die spätere Erarbeitung der Stadtführung wird dadurch das Anfertigen und Präsentieren von Informationsmaterial eingeübt ${ }^{4}$, während die Lerner gleichzeitig einen ersten Überblick über das historische Geschehen, das auch in Café Slavia thematisiert wird, erhalten.

Die Lektüre des Romans sollte parallel dazu zu Hause stattfinden, um eine möglichst selbstständige und freie, d. h. unvoreingenommene, Rezeption zu gewähren, wobei in einem Lesebegleitheft Notizen zu möglichen Motiven sowie zu Verständnisschwierigkeiten oder Unklarheiten gemacht werden sollen. ${ }^{5}$ Diese werden in der dritten Phase gemeinsam besprochen; die Lektüre muss also bis dahin abgeschlossen sein.

Die zweite Phase besteht in der Hinführung zum Roman als Untersuchungsgegenstand und kann in einer Sitzung absolviert werden. Beim Übergang von Filips Gesamtwerk zu Café Slavia werden dem Kurs in einem ersten Schritt zentrale Themen und Leitfragen vorgestellt und diskutiert, die charakteristisch für Filips Romane sind (etwa das Verhältnis der tschechischen und deutschen Kultur und die gemeinsame Geschichte). Anschließend sollen Bezüge zu Filips Biografie hergestellt werden, indem die Lerner an das Vorwissen aus den Kurzpräsentationen

1 Die Aufteilung der Phasen und die entsprechenden Unterrichtseinheiten samt Arbeitsschritten sind zur besseren Übersicht in Form eines tabellarischen Semesterplans dem Anhang beigefügt.

2 Für weitere literaturbezogene Methoden zur Hinführung und Vorentlastung in der Einstiegsphase vgl. Kepser/Abraham 2016: 231f.

3 Homepage Ota Filip. http://www.otafilip.homepage.t-online.de/ (30.08.17).

$4 \mathrm{Zu}$ den Aufgaben und Kompetenzen eines Stadtführers vgl. Harren/Hoffmann (2010: 142f).

5 Erläuterungen zum didaktischen Nutzen von Lesetagebüchern, -mappen o.Ä. finden sich bei Kepser/Abraham (2016: 224). 
anknüpfen. Die Ergebnisse werden in einem Unterrichtsgespräch festgehalten und bilden den Übergang zu einer abschließenden Sequenz, in der die Lehrkraft die Auswahl und die Bedeutung des Romans als Gegenstand der Auslandsgermanistik erläutert und die Lerner unter Nennung der Lernziele auf die folgenden Analysesitzungen vorbereitet. ${ }^{1}$

Die Phasen drei und vier dienen der Vertiefung der Lerninhalte in Form einer Romananalyse. Für die dritte Phase, die Inhaltsanalyse I, sind zwei Sitzungen veranschlagt. Nach einer kurzen Rekapitulation der Handlung werden die Lektürenotizen im Plenum besprochen und Verständnisschwierigkeiten geklärt. Um das Engagement der Lerner zu steigern und die eigenständige Auseinandersetzung mit dem Gegenstand zu gewähren, richtet sich die Textanalyse im Sinne eines ,entdeckenlassenden' Unterrichts vor allem nach den Lerner-Interessen (vgl. Köck 2005: 311). Für die Lehrkraft gilt es aber darauf zu achten, dass die Hauptmotive, die für das Verständnis der Handlung unerlässlich sind, nicht vernachlässigt werden. ${ }^{2}$

Zur Vorbereitung auf die Stadtführung sollen die Lerner anschließend gruppenweise eine Recherche zu gemeinsam ausgewählten Motiven durchführen. Das Ziel ist hier die Einübung literaturwissenschaftlichen Arbeitens, d. h. die Lerner sollen mithilfe geeigneter Sekundärliteratur selbstständig die Bedeutung der Motive erschließen. ${ }^{3}$ Als Anhaltspunkt kann die Lehrkraft auf Untersuchungen zu anderen Werken Filips (z.B. Kubica 2012, Holý 2004) verweisen, auf Nachschlagewerke zu gängigen Motiven in der Literatur (z.B. Daemmrich 1995) oder zur (ostmittel-) europäischen Exilliteratur im Allgemeinen (z.B. Behring et al. 2004). Die Ergebnisse werden wiederum in Kurzpräsentationen festgehalten; die Dauer hängt von der Anzahl der Motive und Gruppen ab. Die Anfertigung kann je nach verbleibender Zeit bereits in der ersten Sitzung der dritten Phase beginnen und in der zweiten Sitzung beendet werden, ist aber auch als Hausaufgabe denkbar. Der Schwerpunkt der zweiten Sitzung liegt in der Präsentation und Besprechung der Ergebnisse.

In der vierten Phase, der abschließenden Sitzung zur Inhaltsanalyse, steht die gemeinsame Erarbeitung der Romanbotschaft und der enthaltenen Kritik im Fokus, die sich - wie gezeigt wurde - im Geschichtsbild niederschlägt. In einem Unterrichtsgespräch soll der Roman hier unter Rückgriff auf das erworbene Wissen aus den Phasen eins und zwei biografisch und realgeschichtlich kontextualisiert werden.

1 Die Begründung und die Lernziele entsprechen denen, die in Punkt 3.1 dargelegt wurden.

2 Gemeint ist hier vor allem das Maskenmotiv, der hospoda-Topos und das damit verbundene Thema des Identitätsverlustes, aber auch die Metaphorik der Stadtbeschreibung, auf die hier nicht eingegangen werden kann.

3 Vgl. hierzu die textkompetenzorientierten Methoden des „kulturhistorischen Lernen[s] durch und mit Literatur" bei Widawska (2015: 149-157) sowie das Phasenmodell zum Verstehen von und zur Arbeit mit literarischen Texten von Kepser/Abraham (2016: 237-238). 
Die Produktionsphase wird mit einer Übergangssitzung eingeleitet, in der die Vorbesprechung des Gruppenprojekts erfolgt. Die Lehrkraft erörtert zum Einstieg mit den Lernern, inwiefern die Stadtführung durch Prag einen Beitrag zur (kulturellen) Annäherung zwischen Tschechien und Deutschland leisten kann. ${ }^{1}$ Danach werden die organisatorischen Rahmenbedingungen des Projekts geklärt: die Einteilung der Gruppen, die Verteilung der Aufgaben sowie die Festlegung von Stationen, einer Route und eines Termins. ${ }^{2}$ Es empfiehlt sich, für jede Station eine zuständige Gruppe zu bilden. Zudem muss abgesprochen werden, wie das Präsentationsmaterial für eine Station gestaltet sein soll. ${ }^{3}$

In der sechsten und längsten Phase findet die eigentliche Erstellung der Stadtführung bzw. des Materials statt. In den hierfür vorgesehenen sechs Sitzungen verfassen die Lerner in Gruppen Informationstexte, erstellen eine Stadtkarte mit den Stationen und gestalten entsprechende Plakate. Zur Förderung selbstständigen Arbeitens bei gleichzeitiger Teamarbeit bietet es sich an, jedem Gruppenmitglied eine eigene Aufgabe zuzuordnen (vgl. Köck 2005: 333f.). Es kann beispielsweise ein Verantwortlicher für realgeschichtliche Informationen gewählt werden, einer für entsprechende Romanpassagen und ein anderer für biografische Bezüge. Nach dem Konzept organisierter Freiarbeit hält sich die Lehrkraft während dieser Phase im Hintergrund, steht den Lernern aber mit Hinweisen zu Quellen, Recherche und Formulierungen zur Seite (vgl. Köck 2005: 304).

Die abschließende, siebte Phase besteht in der Durchführung der Stadtführung. Hier geht es darum, dass die Lerner im Sinne einer interkulturellen Vermittlung das, was sie selbst erarbeitet haben, an Außenstehende weitergeben. Selbstverständlich bietet sich hier auch die Lesung einer Romanpassage, zum Beispiel im Café Slavia, an, zumal beide Verfahren - Vorlesen und Vortragen - sowohl der kommunikativen Kompetenz als auch dem Wissensgewinn dienen (vgl. Kepser/Abraham 2016: 246f.).

Das Publikum setzt sich idealerweise aus einer Gruppe deutscher bzw. deutschsprachiger Interessenten zusammen, beispielsweise einem DaF-Kurs, deutschsprachigen (Gast-)Studenten oder auch Touristen. Alternativ wäre - bei entsprechenden beiderseitigen Sprachkenntnissen - auch eine zweisprachige Durchführung denkbar.

In einem kursinternen Gespräch wird abschließend zusammengetragen, wie die Umsetzung des Projektplans geklappt hat und inwiefern das Ziel, die gewohnte Umgebung und deren Geschichte aus einer neuen Perspektive zu betrachten,

1 Vgl. hierzu auch den Stellenwert der Interaktion in kulturübergreifenden Stadtführungen (Costa 2010: 107-14).

2 Ein Vorschlag für eine mögliche Route bzw. Stationen liegt dem Anhang bei.

3 Man kann den Lernern beispielsweise eine Vorlage zur Verfügung stellen. Ein Muster hierfür wird in Punkt 3.3 dargelegt. 
erreicht wurde. Bestenfalls liegt dafür auch Feedback des Publikums vor, das zum Beispiel mithilfe eines kurzen Fragebogens ermittelt wurde und zuvor gemeinsam festgelegte Kriterien (wie Präsentation, Informationsgehalt und Gestaltung) enthält (vgl. Köck 2005: 316). ${ }^{1}$

\subsection{Beispiel für eine mögliche Station: Brücke der Legionen}

Passend zum zentralen Roman-Thema, dem wechselhaften Geschichtsverlauf, sollen die Lerner zu jeder Station einerseits realgeschichtliche Informationen erarbeiten und andererseits die entsprechende Inszenierung im Roman. Daneben sollen Interpretationsmöglichkeiten für die literarische Darstellungsweise und eventuelle Abweichungen von der Realität thematisiert werden und - sofern vorhanden Bezüge zu Filips Biografie.

Als mögliche Station weist die Brücke der Legionen alle dieser drei Ebenen auf. An ihrem Beispiel wird nun gezeigt, wie die Gegenüberstellung realgeschichtlicher und im Roman enthaltener Informationen inhaltlich gestaltet werden kann. Zur besseren Übersicht wurden die Informationen so, wie sie den Lernern als Vorlage gegeben werden können, tabellarisch festgehalten. ${ }^{2}$ Im Anschluss an die abgebildete Tabelle folgt eine kurze Erläuterung der darin enthaltenden Informationen.

\begin{tabular}{|l|l|}
\hline \multicolumn{2}{|l|}{ Station: Brücke der Legionen } \\
\hline Realgeschichtlicher Hintergrund': & Darstellung im Roman: \\
\hline Erbaut 1839 zur Verbindung der & Belecredos mokiert sich über die \\
Nationalallee mit der Prager Kleinseite & Namensänderungen und hält sich nicht an \\
1839-1919: Franzensbrücke/most císaře & die jeweiligen Bezeichnungen, er verweist \\
Františka I., benannt nach Kaiser Franz I. & stets auf den ursprünglichen Namen \\
1919-1940: Gründung der & („Franzensbrücke““, vgl. CS: 44, 151) \\
Tschechoslowakei, Umbenennung in & Auch der Erzähler (Spaziergänger) kritisiert \\
Brücke der Legionen /Most Legií & die Umbenennungen, er will die Brücke \\
1940-1945: Umbenennung in Smetana- & nicht überqueren und nimmt dafür \\
Brücke/Smetanův most durch die & Umwege in Kauf: "Auf dieser Brücke fühlte \\
nationalsozialistische Besatzung & ich mich nicht wohl. Sie hat auch zu oft \\
1945-1960: Rückkehr zum Namen Brücke & den Namen gewechselt“ (CS: 7) \\
der Legionen mit Ende des 2. Weltkrieges & \\
1960-1990: Umbenennung in Brücke & \\
des 1. Mai/most 1. Máje durch das & \\
sozialistische Regime & \\
Seit 1990 (,Samtene Revolution') wieder & \\
als Brücke der Legionen bezeichnet & \\
\hline
\end{tabular}

1 Für benotete Kurse bedarf es selbstverständlich einer differenzierteren Kriterienauswahl und eines gegenstandsangemessenen Bewertungssystems, das die (von der jeweiligen Kursart abhängigen) zu erwerbenden Kompetenzen abdeckt. Auch in diesem Fall sollten die Lerner in die Festlegung der Kriterien miteinbezogen werden (vgl. Köck 2005: 316).

2 Die biografischen Bezüge werden hier entsprechend Filips Mittlerrolle zwischen Realität und Fiktion in einer Zwischenposition dargestellt. 

Bedeutung: politische Umbrüche führen
zu Umbenennung der Brücke und spiegeln
somit den Verlauf der Geschichte wider
Deutung: Die Thematisierung entspricht Belecredos kritischem Geschichtsbild, nachdem sämtliche Umwälzungen einen Kostüm- oder Maskenwechsel darstellen

Biografischer Bezug:

Filip wohnt von 1945 bis 1968 in Prag und erlebt dort selbst alle politischen Umstürze samt ihren Konsequenzen (Militärdienst, Schreibverbot, Zwangsarbeit) mit (vgl. Filip 2012: 119f.)

Er äußert sich dazu wie folgt: "So lange ich lebe, will ich keine große Geschichte mehr erleben, keine Umstürze, keine Revolutionen, geschweige denn Kriege [...]. Mit achtzig Jahren habe ich sieben Regime, fünfzehn [...] Staatspräsidenten, einen Adolf Hitler [...], einen Stalin [...] erlebt und überlebt“" (Filip 2012: 9)

Deutung: Wie sein Protagonist kritisiert auch Filip den wechselhaften und konfliktlastigen Verlauf der Geschichte, den er selbst miterlebt, und sucht nach einem Ausweg.

Die Brücke der Legionen wird stets aus politischen Gründen umbenannt (vgl. Ledvinka 2007: 338). Realgeschichtlich spiegelt sich also in den Umbenennungen die wechselhafte Geschichte Prags wider. Nach ihrer Fertigstellung 1839 wird sie zu Ehren Kaisers Franz I. Franzensbrücke genannt (vgl. Ledvinka 2007: 338). Mit dem Ende der Habsburgermonarchie und der Gründung der Tschechoslowakei erhält sie bis 1940 den Namen Brücke der Legionen, in Gedenken an die Tschechoslowakischen Legionen im Ersten Weltkrieg (vgl. Ledvinka 2007: 338). Während der Zeit der nationalsozialistischen Besetzung heißt sie Smetana-Brücke (vgl. Ledvinka 2007: 340), wird aber mit deren Ende wieder als Brücke der Legionen bezeichnet (vgl. Ledvinka 2007: 340f.). Das sozialistische Regime benennt sie 1960 in Brücke des 1. Mai um (vgl. Ledvinka 2007: 342), bevor sie 1990 im Zuge der Samtenen Revolution ihren heutigen Namen, Brücke der Legionen, zurückerhält (vgl. Ledvinka 2007: 342).

Wie im Rahmen der Textanalyse gezeigt wurde, ist es eben diese von Umstürzen geprägte Vergangenheit, die Belecredos im Roman kritisiert. Die Umbenennungen sind, genauso wie sein Maskenwechsel, Teil einer komödienhaften Weltgeschichte, in der die Brücke der Legionen eine von vielen Prager Requisiten darstellt. Aus diesem Grund kann bzw. will der Graf die Namensänderungen nicht ernst nehmen und macht sich darüber lustig (vgl. CS: 44, 151), ebenso wie der Erzähler, der die Brücke aus diesem Grund sogar meidet (vgl. CS: 7). Die realen und politisch bedingten Umbenennungen werden im Roman aufgegriffen, um das kritische Geschichtsbild zu verdeutlichen, das Filip mit seinem Protagonisten teilt.

Wie der Graf erlebt auch Filip sämtliche Umbrüche, die zum Namenswechsel der Brücke der Legionen geführt haben, mit. In seinen Verspäteten Abrechnungen resümiert er, er habe sieben Regime, fünfzehn Staatspräsidenten, Hitler sowie Stalin „erlebt und überlebt“ (Filip 2012: 9) und wünsche sich, „[...] dass sie [die 
Geschichte] nicht mehr meine Wege kreuzt, dass sie mich vergisst“ (Filip 2012: 9) - ein Schicksal, dass sich für seinen Antihelden Belecredos erfüllt, der eingemauert und maskiert im Keller der deutschen Botschaft stirbt, unweit der Brücke der Legionen (vgl. CS: 267).

Neben der Brücke der Legionen gibt es zahlreiche weitere Erinnerungsorte und Denkmäler, die auf diese Weise miteinander in Verbindung gebracht werden können, beispielsweise die Karlsbrücke, das Prager Jesulein, der Wenzelsplatz oder auch die Statue des Heiligen Nepomuk. ${ }^{1}$

\section{Fazit und Ausblick}

Das vorliegende Projektkonzept zu Filips Café Slavia kommt den besonderen Anforderungen der Auslandsgermanistik nach: Durch das Aufsuchen zentraler Handlungsorte des Romans sowie der realen Geschichte wird es den Lernern möglich, eben diese aus einer neuen Perspektive zu betrachten. Auf kreative Weise werden die Lerner dazu angeregt, eine Verbindung zwischen der fiktiven Lebensgeschichte und der Kritik an der Realität herzustellen, mit der auch eine Aufarbeitung der problematischen tschechisch-deutschen Vergangenheit einhergeht.

Darüber hinaus sind auch weitere Themenstellungen auf Basis des Romans denkbar: etwa eine Untersuchung der Veränderungen in der tschechischen Ausgabe gegenüber dem deutschen Original oder auch ein Seitenblick auf die Darstellung des Café Slavias in anderen literarischen Werken, z.B. in Jaroslav Seiferts gleichnamigem Gedicht (Seifert 1988) sowie in Rainer Maria Rilkes Erzählungen König Bohusch und Die Geschwister (Rilke 1989).

Filips gesamtes literarisches Schaffen bietet Raum für vielseitige Reflexionen über das Eigene und Fremde, das Vergangene und Künftige, und können damit einen Beitrag zur Auslandsgermanistik leisten, der letztlich auch seinem Anliegen gerecht wird: dem Entdecken von Gemeinsamkeiten, von „verschüttete[n] Grundmauern, [...] auf welchen wir eine gemeinsame Zukunft in Europa bauen können“ (Schmitz 2012: 192).

1 Eine ausführlichere Liste mit möglichen Stationen befindet sich zusammen mit einer entsprechenden Route im Anhang. Sie kann den Lernern gemeinsam mit dem Beispiel der Brücke der Legionen zur Orientierung vorgelegt werden. 


\section{Anhang}

a) Semesterplan für das Projekt einer literarischen Stadtführung in Prag in sieben Phasen

\begin{tabular}{|c|c|}
\hline $\begin{array}{l}\text { Einstieg } \\
\text { (2 Sitzungen) }\end{array}$ & $\begin{array}{l}\text { Erster Überblick über Leben und Werk Ota Filips durch die } \\
\text { Lehrkraft } \\
\text { Gruppen-Kurzreferate (5-10 Minuten) zu den drei } \\
\text { Lebensstationen Ostrau, Prag und München (evtl. mit Karte), } \\
\text { Erarbeitung und Präsentation im Unterricht } \\
\text { [Selbstständige, "freie" Romanlektüre zu Hause mit dem Auftrag, } \\
\text { sich Notizen zu Unklarheiten/Verständnisschwierigkeiten/ } \\
\text { Motiven etc. zu machen, sollte bis spätestens Phase } 3 \\
\text { abgeschlossen sein] }\end{array}$ \\
\hline $\begin{array}{l}\text { Hinführung } \\
\text { (1 Sitzung) }\end{array}$ & $\begin{array}{l}\text { Übergang vom Gesamtwerk Filips zum Roman Café Slavia: } \\
\text { Vorstellung und gemeinsame Besprechung zentraler Themen und } \\
\text { Leitfragen in Filips Gesamtwerk } \\
\text { Lernergeleitete Herstellung von Bezügen zur Biografie durch } \\
\text { Anknüpfung an Vorwissen aus den Kurzreferaten } \\
\text { Erläuterung der Auswahl und Bedeutung des Romans als } \\
\text { Gegenstand der Auslandsgermanistik durch die Lehrkraft }\end{array}$ \\
\hline $\begin{array}{l}\text { Vertiefung/ } \\
\text { Inhaltsanalyse I } \\
\text { (2 Sitzungen) }\end{array}$ & $\begin{array}{l}\text { Kurze, gemeinsame Zusammenfassung des Romaninhalts } \\
\text { Besprechung der Lektüre-Notizen/-Probleme und der } \\
\text { Hauptmotive in Grundzügen (z.B. Identitätsbildung/-verlust: } \\
\text { Masken, Café/hospoda- } \\
\text { Topos) im Plenum } \\
\text { Lerner-Recherche (je nach Zeit: im Unterricht und/oder als } \\
\text { Hausaufgabe) mit Kurzpräsentationen zu vorher ausgewählten } \\
\text { Motiven, im Anschluss Besprechung der Ergebnisse im Unterricht }\end{array}$ \\
\hline $\begin{array}{l}\text { Vertiefung/ } \\
\text { Inhaltsanalyse II } \\
\text { (1 Sitzung) }\end{array}$ & $\begin{array}{l}\text { Gemeinsame Erarbeitung von Roman-Botschaft und enthaltener } \\
\text { Kritik mit Fokus auf das vermittelte Geschichtsbild } \\
\text { Biografische und realgeschichtliche Kontextualisierung des } \\
\text { Romans durch Anknüpfung an Erlerntes aus den Phasen } 1 \text { und } 2 \\
\text { Überleitendes Unterrichtsgespräch zum Gruppenprojekt: } \\
\text { Thematisierung der Intention Filips als, praktische Realisierung' } \\
\text { seines Anliegens in Form einer Stadtführung }\end{array}$ \\
\hline $\begin{array}{l}\text { Übergang zu } \\
\text { Produktion } \\
\text { (1 Sitzung) }\end{array}$ & $\begin{array}{l}\text { Vorbesprechung des Gruppenprojekt: Organisatorisches, } \\
\text { Gruppeneinteilung, Aufgabenverteilung, Hinweise und } \\
\text { Hilfestellung zu Recherche bzw. Quellen durch Lehrkraft, } \\
\text { gemeinsame Festlegung der Route mit Stationen }\end{array}$ \\
\hline
\end{tabular}




\begin{tabular}{|c|c|}
\hline $\begin{array}{l}\text { Produktion } \\
\text { (6 Sitzungen) }\end{array}$ & $\begin{array}{l}\text { Lernergeleitete Planung und Konzipierung einer literarischen } \\
\text { Stadtführung in Prag in Gruppen } \\
\text { Vorschlag zur Arbeitsteilung: eine Gruppe erarbeitet für je eine } \\
\text { Station Informationen zum realgeschichtlichen Hintergrund, zur } \\
\text { Thematisierung im Roman sowie zu biografischen Bezügen (falls } \\
\text { vorhanden) } \\
\text { Anfertigung des Präsentationsmaterials in Gruppen: Schreiben } \\
\text { von Informationstexten, Erstellung einer Stadtkarte mit der Route, } \\
\text { Erstellung von Plakaten, Broschüren o.Ä. für die Teilnehmer } \\
\text { Vorschlag: Gruppeninterne Aufgabenteilung je nach Interesse/ } \\
\text { Fertigkeiten der Lerner } \\
\text { Lehrkraft stellt Vorlage für die inhaltliche Ausarbeitung einer } \\
\text { Station und eine Karte mit einer möglichen Route als Hilfsmaterial } \\
\text { zur Verfügung }\end{array}$ \\
\hline $\begin{array}{l}\text { Umsetzung } \\
\text { (1 Sitzung) }\end{array}$ & $\begin{array}{l}\text { Durchführung der Stadtführung } \\
\text { Weitervermittlung des Gelernten/Erarbeiteten an } \\
\text { "Außenstehende“ (Touristen/andere Studierende) }\end{array}$ \\
\hline
\end{tabular}

b) Mögliche Stationen der Stadtführung und Vorschlag für eine Route 


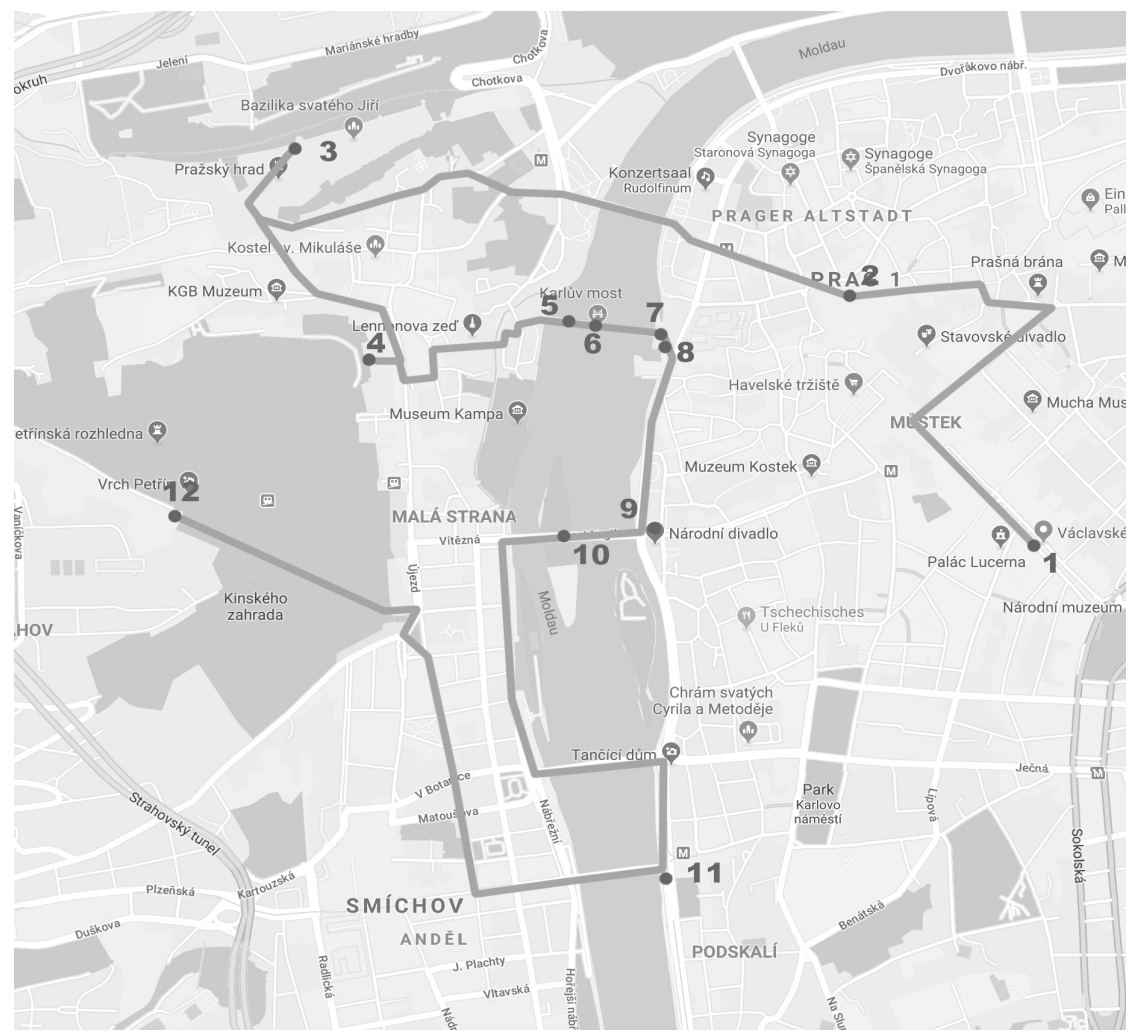

Bildquelle: https://www.google.de/maps/place/Prag,+Tschechien/@50.0593325,14.1854451,10z/data=!3m1!4b1!4m5!3m4!1s0x470b939c0970798b:0 x400afOf66164090!8m2!3d50.0755381!4d14.4378005 (06.09.2017).

1) Wenzelsplatz, Statue Hl. Wenzel

2) St. Niklaskirche

3) St. Veitsdom

4) Maria vom Siege, Prager Jesulein

5) Karlsbrücke

6) Nepomukstatue

7) Altstädter Brückenturm

8) Kreuzherrenplatz, Statue Karl IV.

9) Café Slavia

10) Brücke der Legionen

11) Moldaukai

12) Laurenziberg 


\section{Literaturverzeichnis}

Behring, Eva / Brandt, Juliane / Dózsai, Mónika / Kliems, Alfrun / Richter, Ludwig / Trepte, Hans-Christian (2004): Grundbegriffe und Autoren ostmitteleuropäischer Exilliteraturen 1945-1989. Ein Beitrag zur Systematisierung und Typologisierung. Hrsg. von Eva Behring, Alfrun Kliems und Hans-Christian Trepte. Stuttgart, Franz Steiner (= Forschungen zur Geschichte und Kultur des östlichen Mitteleuropa 20).

Costa, Marcella (2010): Stadtführungen unter der Bedingung von Fremdheit und Fremdsprachigkeit. In: Costa, Marcella / Müller-Jacquier, Bernd (Hg.): Deutschland als fremde Kultur: Vermittlungsverfahren in Touristenführungen. München, Iudicium (= Reihe interkulturelle Kommunikation 9). S. 96-117.

Daemmrich, Horst S. (Hg.) (1995): Themen und Motive in der Literatur. Ein Handbuch. Tübingen, Francke.

Faryar, Massum (2005): Fenster zur Zeitgeschichte - Eine monographische Studie zu Ota Filip und seinem Werk. Berlin, Mensch \& Buch.

Filip, Ota (2001): Café Slavia. München, Herbig.

Filip, Ota (2012): Verspätete Abrechnungen. Dresden, Thelem/Eckhard Richter.

Grucza, Franciszek (1997): Mitteleuropa - Deutsch - Auslandsgermanistik. In: Jahrbuch Deutsch als Fremdsprache 23/1997. S. 297-314.

Harren, Inga / Hoffmann, Wiltrud (2010): Wissen vermitteln, Interesse wecken, Disziplin erhalten: interaktive Verfahren in einer fremdsprachigen Stadtführung mit Jugendlichen. In: Costa, Marcella / Müller-Jacquier, Bernd (Hg.): Deutschland als fremde Kultur: Vermittlungsverfahren in Touristenführungen. München, Iudicium (= Reihe interkulturelle Kommunikation 9). S. 141-162.

Historie Café Slavia. https://www.cafeslavia.cz/de/uber-uns/historie/ (16.08.2017).

Holý, Jiř́ (2004): Ota Filips Lojzek-Lapáček-Roman. In: Holý, Jiř́ / Zand, Gertraude (Hg.): Transfer. Vyhnániodsun v kontextu české literatury. Brünn, Host. S. 107-124.

Homepage Ota Filip. http://www.otafilip.homepage.t-online.de/ (30.08.17).

Kepser, Matthis / Abraham, Ulf (2016): Literaturdidaktik Deutsch. Eine Einführung. Berlin, Erich Schmidt (= Grundlagen der Germanistik 42).

Kersten, Sandra / Schenke, Manfred Frank (Hg.) (2005): Spiegelungen. Entwürfe zu Identität und Alterität. Festschrift für Elke Mehnert. Berlin, Frank \& Timme.

Kliems, Alfrun (2002): Im Stummland. Zum Exilwerk von Libuše Moniková, Jiř̀ Gruša und Ota Filip. Frankfurt a.M., Peter Lang (= Europäische Hochschulschriften: Slawische Sprachen und Literaturen 67).

Köck, Peter (2005): Handbuch der Schulpädagogik für Studium, Praxis, Prüfung. Donauwörth, Auer.

Kubica, Jan (2013): Die Nachkriegszeit im Werk von Ota Filip. In: Slezský sbornik: Acta Silesiaca 111/1/2013. S. 107-120.

Kubica, Jan (2012): Spisovatel Ota Filip. Brno, Větrné Mlýny.

[Art.] Hospoda in: Langenscheidt Digital: Tschechisch - Deutsch Wörterbuch. München, Langenscheidt.

Ledvinka, Václav (2007): Die Namen von Prager öffentlichen Räumen als Spiegelung des Wandels der politischen Realität im 20. Jahrhundert. In: Jaworski, Rudolf (Hg.): Die Besetzung des öffentlichen Raumes. Politische Plätze, Denkmäler und Straßennamen im europäischen Vergleich. Berlin, Frank \& Timme. S. 337-344.

Rilke, Rainer Maria (1989): König Bohusch. Geschichten aus Prag. Berlin, Verlag der Nation. 
Schmitz, Walter (2012): Fluchtversuche eines Geschichtenerzählers. Ota Filips tschechischdeutsche Autorschaft. In: Filip, Ota (2012): Verspätete Abrechnungen. Dresden, Thelem/ Eckhard Richter. S. 151-216.

Seifert, Jaroslav (1988): Der Halleysche Komet. Gedichte. Aus dem Tschechischen von Franz Peter Künzel. München, Piper.

Widawska, Barbara (2015): Gedächtnis - Geschichte - Literatur. Zum literarischen Umgang mit der Zeitgeschichte. Ein integratives Konzept für den DaF-Unterricht im Germanistikstudium in Polen. Hamburg, Dr. Kovac (= Studien zur Germanistik 57).

Julia Enzinger

Universität Regensburg

julia.enzinger@gmx.de 